\title{
Pediatric CT of the Lung: Influences on Image Quality
}

\author{
Enno Stranzinger ${ }^{1 *}$, Sebastian Tobias Schindera ${ }^{1}$, Jennifer Larissa Cullmann ${ }^{1}$, \\ Ralph Herrmann ${ }^{1}$, Shu-Fang Hsu Schmitz ${ }^{2}$, Rainer Wolf ${ }^{1}$ \\ ${ }^{1}$ Department of Diagnostic, Interventional and Pediatric Radiology, Bern University Hospital, Bern, Switzerland \\ ${ }^{2}$ Institute of Mathematical Statistics and Actuarial Science, University of Bern, Bern, Switzerland \\ Email: "enno.stranzinger@insel.ch
}

Received November 27, 2012; revised December 28, 2012; accepted January 5, 2013

\begin{abstract}
Objective: To assess influential factors of CT on image quality of the lung in children. Materials and methods: Retrospective evaluation of 82 consecutive chest-CT-scans in 50 children (1 - 16 years, 17 females and 33 males). Two pediatric radiologists evaluated in consensus the subjective image quality on lung windows using a 4-point scale $(1=$ very good, 2 =good, 3 = moderate, 4 = poor). Ventilation, motion artifacts and beam hardening artifact were included in this score. The effects of the following factors were evaluated: 1) CT-settings (tube energy, tube current); 2) Patient's age, weight, chest size, ventilation; 3) Artifacts of devices, tubes and lines; 4) Combination MRI of the abdomen prior to CT of the chest with the same sedation/anesthesia in oncological patients. Results: The odds of having a better image quality increase with patient's age, weight and chest diameter in a multiple-factor model. There was no difference between tube current protocols. In infants $(<15 \mathrm{~kg}$ ) subjective image quality was good in only $1(8 \%)$, moderate in $8(67 \%)$ and poor in $3(25 \%)$ scans. In childhood and adolescence $(15-90 \mathrm{~kg}) 25(36 \%)$ scans were very good, $28(40 \%)$ good, 15 $(21 \%)$ moderate and $2(3 \%)$ poor. Artifacts of tubes and lines have no statistical significant influence on image quality. Lower lung densities were related to better ventilation and older children. Conclusion: Increasing dose parameters may not increase necessarily subjective image quality in infants $(<15 \mathrm{~kg})$, rather than good ventilation, optimal preparation and avoiding artifacts. A possible explanation of the rather moderate image quality in infants may be the alveolar stage of the lung. Up to two years of age the lung has a high specific lung volume per $\mathrm{kg}$ and a low total lung volume with a low alveolar surface.
\end{abstract}

Keywords: CT; Lung; Image Quality; Children

\section{Introduction}

Computed tomographies (CT) of the pediatric chest continue to be an increasingly used imaging modality. Particularly in oncological patients, but also in children with cystic fibrosis study-protocols propose chest-CT-scans on a regular basis even for very young children. Babies and young children under 2 years of age are a challenge to image with CT. There is lack of sufficient compliance and also the fact of the still developing alveoli with a relatively dense lung structure and less aerated lung compared to older children. There is an influence of the sedation to the state of ventilation and atelectasis occur frequently even with optimal hyperventilation before the scan. In children under 6 years of age, CT scans are possible in normal breathing children, but there is often need of mild sedation during the application of contrast media and the scan. The advantages of CT are high resolution of small lung nodules, the possibility of describing bronchiectasis, mucus plugging, inflammation, air trapping

${ }^{*}$ Corresponding author. with an optimal contrast between air and lung parenchyma. CT-scans have an overall better availability and less need for sedation or anesthesia compared with MRIscans. Disadvantages remain radiation dose and sedation in younger children.

The newborn lung has completed the saccular stage with development of the terminal air sacs. During the alveolar stage (36 weeks to infancy), the size and number of alveoli increase. At birth 100 million alveoli are estimated, postnatal alveolar development continues to the mature lung with 300 million alveoli. The lung volume increases from $200 \mathrm{ml}$ to $5 \mathrm{~L}$ and the lung weight from $50 \mathrm{~g}$ to $800 \mathrm{~g}$. The alveolar diameter increases from $150 \mu \mathrm{m}$ to $300 \mu \mathrm{m}$ [1].

All these influences on lung maturation and growth may have an effect on CT-protocols and expected image quality.

Many authors described the ALARA (as low as reasonable achievable) principle in pediatric CT-scans, strategies and low dose techniques of CT in children [2-6]. Lee et al. and Siegel recommended $80 \mathrm{kVp}$ protocols with the lowest possible mAs for aortic anomalies, thoracic vessels and airways in the pediatric population up 
to $50 \mathrm{~kg}[7,8]$. Whereas Cody et al. found beam-hardening artifacts when pediatric CT examinations were performed with $80 \mathrm{kVp}$ and they suggested the use of 100 $120 \mathrm{kVp}$ in this population [9]. Rogalla and Stöver proposed to adjust the mAs setting for $120 \mathrm{kVp}$ protocols based on the patients weight $(\mathrm{mAs}=\mathrm{kg}+5)$ to reduce radiation dose $[10,11]$. Newer CT-scanners use iterative reconstruction techniques and improve the image quality in very low dose CT-protocols.

The physiological changes of the lung play a major role in imaging the lung especially in younger infants. It remains a challenge to adapt $\mathrm{CT}$-settings to the needs of the clinical question (lung nodule detection, air trapping, bronchiectasis etc.) and to generate good quality images with the lowest dose possible. Optimal ventilation of the lungs in this age group is a major problem. Close collaboration with anesthesiologist and or pneumologists is important for a good preparation with sufficient aeration of the lung and avoiding atelectasis and also artifacts from lines, ECG-electrodes and devices.

The purpose of our study was to assess effects for different sized children and other influential factors on image quality in pediatric chest CT-scans to optimize image quality in low dose protocols.

\section{Materials and Methods}

Retrospective evaluation of 82 consecutive chestCT-scans (64 rows Somatom Sensation Cardiac, Siemens, Forchheim, Germany) in 50 children (1 - 16 years, 17 females, 33 males) during a two year period. High resolution computed tomography (HRCT) was not included in this study (Table 1). Two board-certified pediatric radiologists with 5 and 10 years of experience with pediatric chest $\mathrm{CT}$ evaluated in consensus the subjective image quality on lung windows with $3 \mathrm{~mm}(<20-30 \mathrm{~kg})$ or 5 $\mathrm{mm}(>20-30 \mathrm{~kg})$ slice thickness using a 4-point scale $(1=$ very good, 2 = good, $3=$ moderate, $4=$ poor) (Figures 1-4). The subjective image quality assessment was

Table 1. Characteristics of 82 scans (STD = standard deviation).

\begin{tabular}{|c|c|c|c|c|c|}
\hline Categorical Factor & Category & Nr. scans & Continuous Factor & Statistics & Value \\
\hline \multirow[t]{4}{*}{ Weight (kg) } & $<15$ & 12 & Weight (kg) & Mean & 28.2 \\
\hline & $15-30$ & 39 & & STD & 15.4 \\
\hline & $>30$ & 31 & & Median & 26 \\
\hline & & & & Range & $6.7-90$ \\
\hline \multirow[t]{4}{*}{ Tube energy $(\mathrm{kVp})$} & 80 & 3 & & & \\
\hline & 100 & 68 & Age (years) & Mean & 7.9 \\
\hline & 120 & 11 & & STD & 4.5 \\
\hline & & & & Median & 7 \\
\hline \multirow[t]{3}{*}{ Gender } & M & 42 & & Range & $1-16$ \\
\hline & $\mathrm{F}$ & 40 & & & \\
\hline & & & Average image noise (HU) & Mean & 15.1 \\
\hline \multirow[t]{3}{*}{ Endotracheal tube } & Yes & 13 & & STD & 3.3 \\
\hline & No & 69 & & Median & 14.3 \\
\hline & & & & Range & $9.6-24$ \\
\hline \multirow[t]{3}{*}{ ECG-electrodes } & Yes & 7 & & & \\
\hline & No & 75 & Lateral chest diameter (mm) & Mean & 225.0 \\
\hline & & & & STD & 43.6 \\
\hline \multirow[t]{2}{*}{ Port a cath } & Yes & 35 & & Median & 216 \\
\hline & No & 47 & & Range & $127-329$ \\
\hline Adapted Rogalla formula used & Yes & 52 & mAs tube current & Mean & 37.6 \\
\hline \multirow[t]{2}{*}{$(\mathrm{mAs}=\mathrm{kg}+5)$ for $100 \mathrm{kVp}$ protocols } & No & 30 & & STD & 18.2 \\
\hline & & & & Median & 31.5 \\
\hline Atelecatsis & No & 65 & & & \\
\hline \multirow[t]{2}{*}{ Slice thickness (mm) } & 3 & 53 & & & \\
\hline & 5 & 29 & & & \\
\hline
\end{tabular}




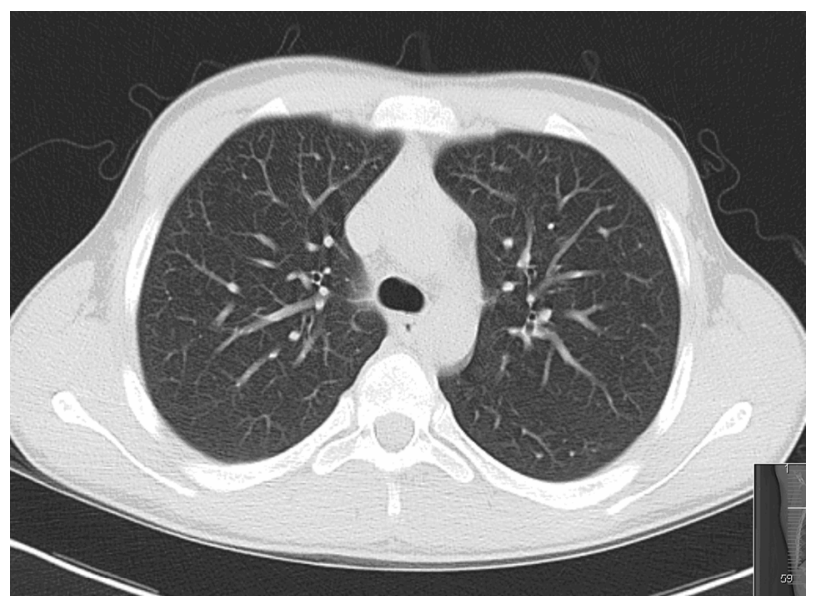

Figure 1. Very good (1) subjective image quality. Patient with $65 \mathrm{~kg}, 100 \mathrm{kVp}$ and $70 \mathrm{mAs}$.

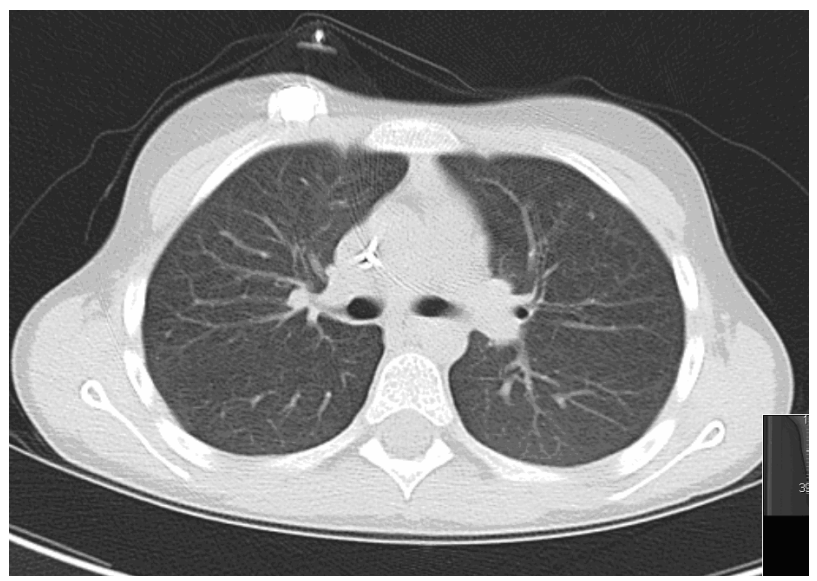

Figure 2. Good (2) subjective image quality with beam hardening artifact of a Port-a-cath. Patient with $35 \mathrm{~kg}, 100 \mathrm{kVp}$ and $38 \mathrm{mAs}$.

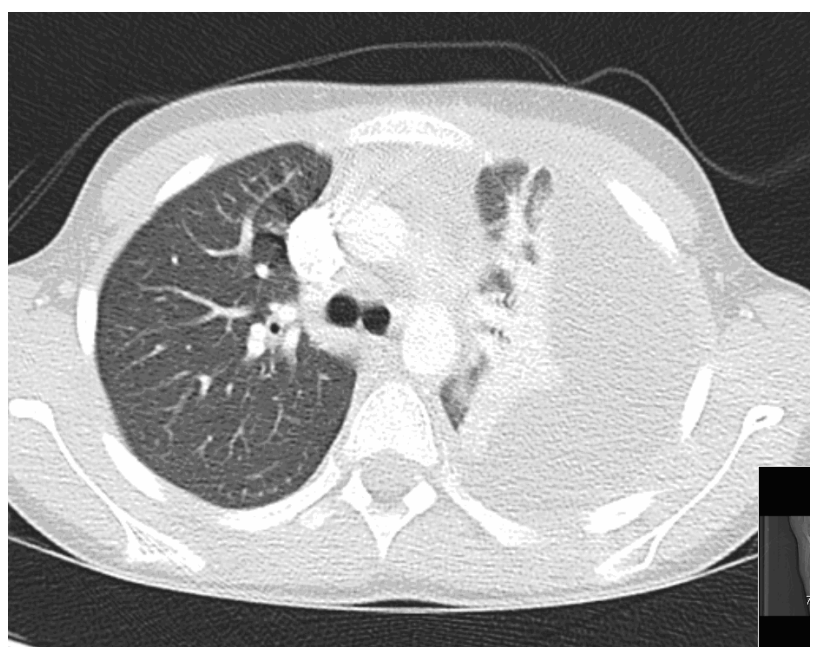

Figure 3. Moderate (3) subjective image quality. Beam-hardening artifact and background noise from contrast media. Large left pleural effusion is noted. Patient with $26 \mathrm{~kg}, 100$ kVp and 35 mAs.

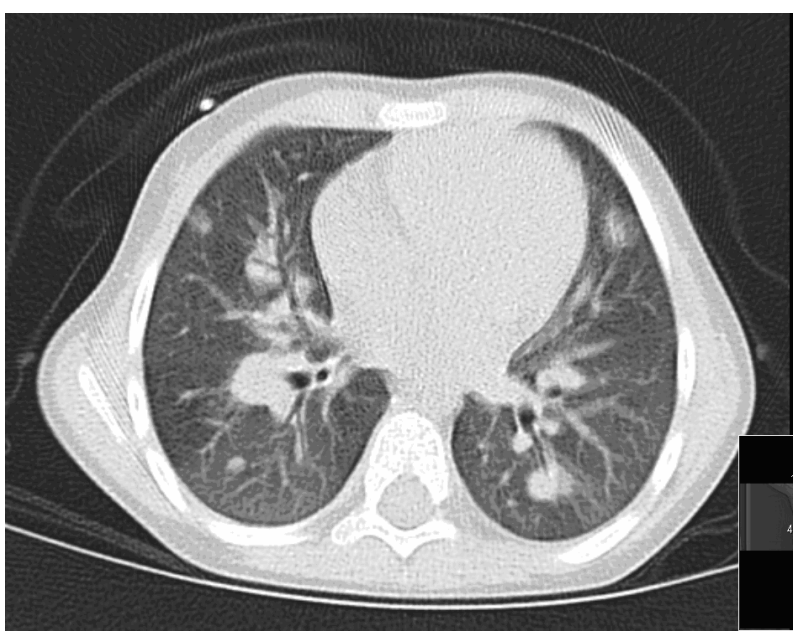

Figure 4. Poor (4) subjective image quality. Low inspiration, motion artifact and beam hardening artifact. Patient with common ALL and EBV associated lymphoproliferative syndrome. Multiple lung nodules are noted. Patient with $13 \mathrm{~kg}$, $100 \mathrm{kVp}$ and $17 \mathrm{mAs}$ (DLP 17).

based on motion artifacts, hardware artifacts, beam hardening artifacts, overall image impression of the lung parenchyma for the evaluation of small lung nodules and pathological changes of the lung parenchyma. The readers were blinded for dose settings, age and indication.

The effects of the following factors on subjective image quality were evaluated: tube energy, average image noise (Hounsfield Unit (HU)) measured in three largest muscle regions at the area of the xyphoid bone, modified Rogalla formula (RF) $(\mathrm{mAs}=\mathrm{kg}+5( \pm 5))$, weight group, indication, dose length product (DLP), endotracheal tube, ECG-electrodes, and chest diameter.

The odds ratio of having better subjective image quality in a single-factor (univariate) analysis was evaluated on each factor using a nonlinear mixed effects model with a cumulative logit link function (proportional odds) for the ordinal response variable (subjective image quality) and a random effect for patient to account for potential correlation between repeated CT-scans within patients. A multiple-factor (multivariate) analysis using the same model was performed after backward selection procedure. Comparisons between groups for continuous outcome variables, e.g. noise and weight, were performed using a linear mixed effects model with a random effect for patient. All analyses were carried out using SAS version 9.1 for Windows (SAS Institute Inc., Cary, NC, USA).

\section{Results}

Noise and subjective image quality:

The average image noise in $80 \mathrm{kVp}(\mathrm{n}=3$ scans) protocols was $21.7 \mathrm{HU}$ (standard deviation (STD) $2.0 \mathrm{HU}$, range, $20.3 \mathrm{HU}-24 \mathrm{HU})$, in $100 \mathrm{kVp}$ ( $\mathrm{n}=68$ scans) 15.4 
HU (STD 2.9 HU, range, $10 \mathrm{HU}-22.6 \mathrm{HU}$ ) and in 120 $\mathrm{kVp}(\mathrm{n}=11$ scans) $11.9 \mathrm{HU}$ (STD $2.5 \mathrm{HU}$, range, 9.6 $\mathrm{HU}-18.3 \mathrm{HU})$. The difference in average image noise across the $3 \mathrm{kVp}$ protocols was statistically significant $(\mathrm{P} \leq$ $0.0006)$ with the tendency for less noise under greater tube energy. In $100 \mathrm{kVp}$ protocols (68 scans), the average image noise in the weight group $<15 \mathrm{~kg}, 15-30 \mathrm{~kg}$ and $>30 \mathrm{~kg}$ was $14.8,15.6$ and $15.3 \mathrm{HU}$, respectively (Table 2), and the difference was not statistically significant.

The average subjective image quality in $80 \mathrm{kVp}$ (mean weight 19.3, STD 14.5) was 3 (STD 1). In $100 \mathrm{kVp}$ protocols the difference in average subjective image quality was statistically significant across weight groups $(<15$, $15-30,>30 \mathrm{~kg})(\mathrm{P} \leq 0.011)$, with the tendency for better image quality at heavier weight or larger chest diameter (Table 2).

In $120 \mathrm{kVp}$ protocols (mean weight 38.8, STD 14.1) the average subjective image quality was 1.4 (STD 0.7 ).

In the single-factor model the odds of having a better subjective image quality increase significantly $(\mathrm{P}<0.05)$ with tube energy, slice thickness, less average image noise, chest diameter, AP chest diameter in $\mathrm{mm}$, tube current mAs, dose length product (DLP), patient's age and patient's weight (Table 3). Specifically, in infants $(<15 \mathrm{~kg})$ subjective image quality was good in only $1 \mathrm{scan}$
$(8 \%)$, moderate in 8 scans $(67 \%)$ and poor in 3 scans (25\%). In childhood and adolescence $(15-90 \mathrm{~kg}) 25$ scans $(36 \%)$ were very good, 28 scans $(40 \%)$ good, 15 scans $(21 \%)$ moderate and 2 scans $(3 \%)$ poor. There was no statistically significant difference in subjective image quality between RF and no RF (Table 3).

In the multiple-factor model the odds of having a better subjective image quality significantly $(P<0.05)$ increase with patient's chest diameter, no prior MRI and slice thickness. Most of the significant factors identified in the single-factor model are no longer significant here, probably due to the association among them. For instance slice thickness, lateral chest diameter, AP chest diameter, tube current, mAs, dose length product (DLP) and pa tient's weight are naturally associated with patient's age;

Table 2. Subjective image quality ${ }^{*}$ and image noise (HU) in $100 \mathrm{kVp}$ protocols. * $(1$ = very good, 2 = good, 3 = moderate, 4 = poor).

\begin{tabular}{lllc}
\hline & $<\mathbf{1 5} \mathbf{~ k g ~ n}=\mathbf{1 5}$ & $\mathbf{1 5}-\mathbf{3 0} \mathbf{~ k g ~ n = 2 1}>\mathbf{3 0} \mathbf{~ k g ~ n}=\mathbf{3 2}$ \\
\hline $\begin{array}{c}\text { image noise (HU): } \\
\text { mean (STD) }\end{array}$ & $14.8(3.2)$ & $15.6(2.6)$ & $15.3(3.3)$ \\
$\begin{array}{c}\text { subjective image } \\
\text { quality: mean (STD) }\end{array}$ & $3.1(0.6)$ & $2.4(0.8)^{*}$ & $1.5(0.6)^{*}$ \\
${ }^{*} \mathrm{P} \leq 0.011$. & & & \\
\end{tabular}

Table 3. Nonlinear mixed effect analysis of subjective image quality on individual factor (OR = odds ratio; OR LCL = lower limit of the $95 \%$ confidence interval for OR; OR UCL = upper limit of the $95 \%$ confidence interval for OR).

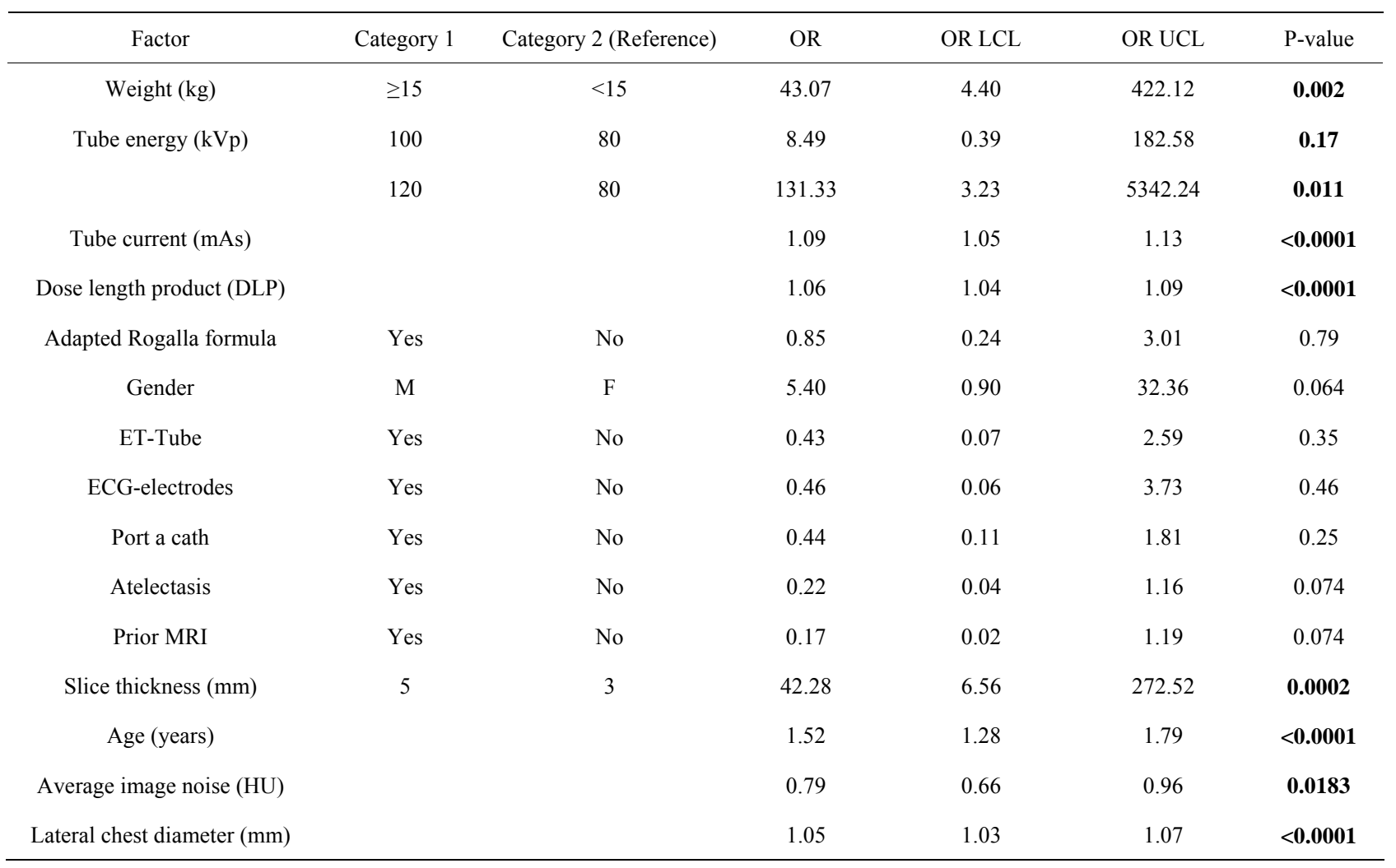


and average image noise is associated with tube energy. On the other hand, prior MRI status was not significant in the single-factor model, but becomes significant when chest diameter and slice thickness are included in the multiple-factor model.

\section{Discussion}

Image contrast is related to the energy of the x-ray photons, equipment filtration and patient size. Increasing the peak kilovoltage reduces the image contrast. Structures with high intrinsic contrast, such as bone and vessels containing iodinated contrast media may be better appreciated at lower tube voltage [4]. This may be used with $80 \mathrm{kVp}$ in infants to image vascular malformations or vascular rings, congenital malformations $(\mathrm{CDH})$, sequester and bone malformations [6,7]. The lowest possible mAs is dependent and limited based on the scout and patient's weight on many CT-scanners. In $80 \mathrm{kVp}$ settings, the mAs need to be higher than in $100 \mathrm{kVp}$ and $120 \mathrm{kVp}$ protocols to obtain the same image noise. In children up to two years, the alveolar stage has a high specific lung-volume per $\mathrm{kg}$ and a low total lung volume with a low alveolar surface. This may cause a low subjective image quality in younger children with either 80 $\mathrm{kVp}$ or $100 \mathrm{kVp}$ protocols.

The subjective image quality with the adapted RF in $100 \mathrm{kVp}$ protocols may be reliable in children over $30 \mathrm{~kg}$ assumed that external artifacts were reduced (ECG electrodes, lines, tubes), good ventilation was achieved and no prior MRI-scan took place (atelectatic changes).

CT-protocols should be easy to perform for the technicians regarding the different weight, age, size and compliance of the children. In hospitals with no primary pediatric experience (emergency situations) guidelines (color codes for age and weight) are helpful to avoid adult protocols and relatively high radiation doses for children (Table 4). Most indications in infants $(<15 \mathrm{~kg})$ may be imaged on MRI (vascular malformations, congenital malformations), since these patients usually need anesthetic sedation. In pediatric chest CT-scans $>$ $75 \mathrm{~kg} 120 \mathrm{kVp}$ protocols may be useful with a weight adapted mAs.

Limitations of the study were the retrospective nature. The number of $80 \mathrm{kVp}$ and $120 \mathrm{kVp}$ protocols was

Table 4. Pediatric chest CT-protocols (64 row CT).

\begin{tabular}{cccc}
\hline & $\mathbf{3} \mathbf{- 3 0} \mathbf{~ k g}$ & $>\mathbf{3 0 ~} \mathbf{~ g g}$ & $>\mathbf{7 5} \mathbf{~ k g}$ \\
\hline $\mathrm{kVp}$ & $80^{*} / 100$ & 100 & 120 \\
$\mathrm{mAs}$ & 30 & $=\mathrm{kg}+5$ or \#CD & $=\mathrm{kg}$ or \#CD \\
$\mathrm{CTDIVol}$ & $0.5^{*} / \leq 1.2 \mathrm{mGy}$ & $\leq 1.5 \mathrm{mGy}$ & $\leq 3 \mathrm{mGy}$ \\
\hline
\end{tabular}

*For vascular malformations, bronchial tree; \#CD = Care Dose for CT scanner with iterative reconstructions. too small for separate statistical analyses. The spare data structure with partial repeated measurements within patients resulted in large variation in estimation, therefore for some factors the confidence intervals for odds ratio were very wide. Also the number of children below 15 $\mathrm{kg}$ was too small to evaluate the optimal $\mathrm{mAs}$ and $\mathrm{kVp}$ settings.

\section{Conclusions}

Increasing dose parameters in infants may not increase necessarily image quality in pediatric $(<15-30 \mathrm{~kg})$ chest CT-scans. It is more important to obtain a good ventilation in younger children (under 6 years of age), avoiding artifacts and also plan the timing of the study before an MRI-scan and not after the MRI scan because of atelectatic changes. In children $<15 \mathrm{~kg}$ it remains difficult with $100 \mathrm{kVp}$ and particularly with $80 \mathrm{kVp}$ protocols to obtain sufficient image quality for the lung parenchyma. A potential explanation for a low subjective image quality in younger children is the alveolar stage in infants up to two years having a high specific lung-volume per $\mathrm{kg}$ and a low total lung volume with a low alveolar surface.

For vascular, osseous or bronchial tree abnormalities, $80 \mathrm{kVp}$ protocols are reliable for infants. In children $>30$ $\mathrm{kg}$ with optimal preparation $100 \mathrm{kVp}$ protocols using weight adapted mAs $(=\mathrm{kg}+5)$ have sufficient subjective image quality for most indications.

In newer generation computed tomography iterative reconstruction algorithm and automatic dose modulation may additionally improve image quality and reduce radiation dose.

\section{REFERENCES}

[1] W. A. Hudson, "Normal and Abnormal Structural Development of the Lung in Polin RA," Fetal and Neonatal Physiology, 2nd Edition, W. B. Saunders, Philadelphia, 1988, p. 1037.

[2] A. Paterson, D. P. Frush and L. F. Donnelly, "Helical CT of the Body: Are Settings Adjusted for Pediatric Patients?" American Journal of Roentgenology, Vol. 176, No. 2, 2001, pp. 297-301.

[3] K. J. Strauss, M. J. Goske, S. C. Kaste, et al., "Image Gently: Ten Steps You Can Take to Optimize Image Quality and Lower CT Dose for Pediatric Patients," American Journal of Roentgenology, Vol. 194, No. 4, 2010, pp. 868873. doi:10.2214/AJR.09.4091

[4] T. L. Slovis, "The ALARA (as Low as Reasonably Achievable) Concept in Pediatric CT Intelligent Dose Reduction," Multidisciplinary Conference Organized by the Society of Pediatric Radiology, Pediatric Radiology, Vol. 32, No. 4, 2010, pp. 217-313.

[5] A. Paterson and D. P. Frush, "Dose Reduction in Paediatric MDCT: General Principles," Clinical Radiology, Vol. 62, No. 6, 2007, pp. 507-517. 


\section{doi:10.1016/j.crad.2006.12.004}

[6] P. Vock, "CT Radiation in Children: Consequences of the American Discussion for Europe," Radiologe, Vol. 42, No. 9, 2002, pp. 697-702.

doi:10.1007/s00117-002-0812-4

[7] E. Y. Lee, M. J. Siegel, C. F. Hildebolt, F. R. Gutierrez, S. Bhalla and J. H. Fallah, "MDCT Evaluation of Thoracic Aortic Anomalies in Pediatric Patients and Young Adults: Comparison of Axial, Multiplanar, and 3D Images," American Journal of Roentgenology, Vol. 182, No. 3, 2004, pp. 777-784.

[8] M. J. Siegel, "Multiplanar and Three-Dimensional MultiDetector Row CT of Thoracic Vessels and Airways in the Pediatric Population," Radiology, Vol. 229, 2003, pp.
641-650. doi:10.1148/radiol.2293020999

[9] D. D. Cody, D. M. Moxley, K. T. Krugh, J. C. O’Daniel, L. K. Wagner and F. Eftekhari, "Strategies for Formulating Appropriate MDCT Techniques When Imaging the Chest, Abdomen, and Pelvis in Pediatric Patients," American Journal of Roentgenology, Vol. 182, No. 4, 2004 , pp. 849-859.

[10] P. Rogalla, B. Stöver, et al., "Low-Dose Spiral CT: Applicability to Paediatric Chest Imaging," Pediatric Radiology, Vol. 29, No. 8, 1999, pp. 565-569. doi: $10.1007 / \mathrm{s} 002470050648$

[11] B. Stöver and P. Rogalla, "CT Examinations in Children," Der Radiologe, Vol. 48, No. 3, 2008, pp. 243-248. doi:10.1007/s00117-007-1600-y 